\title{
EDITORIAL
}

\section{New year, new improvements}

\author{
Hypertension Research (2010) 33, 1-3; doi:10.1038/hr.2009.196
}

$\mathrm{W}$

elcome to the first 2010 issue of Hypertension Research. Last year Hypertension Research underwent many impressive developments, both inside and outside the journal. In January, the journal published the Japanese Society of Hypertension Guidelines for the Management of Hypertension (JSH 2009); new Commentary articles were introduced in August; and advanced online publication (AOP) of articles on nature.com also commenced. These developments, I am sure, must have made the journal look very different. I am very grateful to all those who helped us implement them, as always, the active support of authors, reviewers and readers is indispensible.

Hypertension Research continues to move forward in 2010 with more exciting changes. The first is the introduction of Review Seriessmall collections of review articles on specific topics of recent interest. Three Review Series are scheduled for 2010: '(Pro)Renin Receptors', which appears in this issue; 'Diurnal Variation in Blood Pressure: Clinical Implication and Pathogenesis'; and 'Salt Intake and Inflammation in Hypertension'. We hope you enjoy reading these review articles throughout the year.

Abe, Isao

Abe, Michiaki

Adachi, Hisashi

Aizawa, Yoshifusa

Akasaka, Takashi

Akazawa, Hiroshi

Akiguchi, Ichiro

Akimoto, Tetsu

Akishita, Masahiro

Anan, Ryuichiro

Ando, Katsuyuki

Anzai, Toshihisa

Aoki, Hiroki

Araki, Shin-Ichi

Arima, Hisatomi

Arima, Shuji

Armando, Ines

Asakura, Masanori

Asayama, Kei

Ashida, Terunao

Azuma, Hiroshi

Babazono, Tetsuya

Bader, Michael

Bagi, Zsolt

Baum, Michel

Bechara, Luiz Roberto Grassman

Ben-Dov, Iddo
Bernini, Giampaolo

Bernstein, Kenneth

Brun, Jean-Frederic

Brunini, Tatiana

Calhoun, David

Campese, Con Vito

Campos, Ruy

Cebová, Martina

Ceral, Jiri

Chade, Alejandro

Chang, Nen-Chung

Chappell, Mark

Chen, Elizabeth

Chen, Yuanyuan

Cheng, Xian Wu

Cho, Kyung Woo

Chuang, Yao-Chung

Cicala, Carla

Ciccarelli, Leonardina

Cicero, Arrigo

Coca, Antonio

Cohen, Debbie

Cottone, Santina

Cuneo, Bettina

Daimon, Masao

Dan, Qinghong

Davidson, Michael
Although Hypertension Research will continue to make changes, the journal maintains the same high-quality editorial and publishing practices. Our primary goal is to improve the journal and make it more attractive to readers and authors. Through these developments, we sincerely hope that Hypertension Research can better serve readers as an important source of information and an exciting forum for scientific communication.

We wish you all a very happy and prosperous new year.

\section{ACKNOWLEDGEMENTS}

The Editor-in-Chief, on behalf of the Hypertension Research Editorial Board and the Japanese Society of Hypertension, gratefully acknowledges the generous support from peer reviewers in carefully assessing manuscripts under consideration. The following individuals provided reviews of papers submitted to Hypertension Research from 1 October 2008 to 30 September 2009:

\section{Masatsugu Horiuchi Editor-in-Chief \\ Hypertension Research}

De Gennaro Colonna, Vito

De Paepe, Boel

Delanghe, Joris

Derosa, Giuseppe

Deter, Hans-Christian

Dohi, Yasuaki

Dolan, Eamon

Dominguez, Jesus

Egashira, Kensuke

Egido, Jesus

Eguchi, Kazuo

England, Lucinda

Erez, Offer

Eto, Masaaki

Evans, John

Farsang, Csaba

Ferrari, Paolo

Ferreira, Sandra

Fisher, Naomi

Friso, Simonetta

Fujimura, Akio

Fujio, Yasushi

Fukamizu, Akiyoshi

Fukuda, Michio

Fukuda, Noboru

Fukuo, Keisuke
Frangogiannis, Nikolaos
Funke-Kaiser, Heiko

Galderisi, Maurizio

Gao, Lie

Gaussem, Pascale

Gibson, K.J.

Gillett, Melissa

Godin-Ribuot, Diane

Goto, Atsuo

Grassi, Guido

Grossman, Ehud

Grunberger, George

Hage, Fadi

Halimi, Jean-Michel

Hama, Seiji

Hamed, Sherifa

Hamet, Pavel

Hansen, Dorte

Hasebe, Naoyuki

Hashimoto, Junichiro

Hatazawa, Jun

Hattori, Yoshiyuki

Hayano, Junichiro

Hayashi, Koichi

Hayashi, Matsuhiko

Hayashi, Toshio

Hayden, Pete
Hashimoto, Masayoshi 
Head, Geoffrey

Hermida, Ramon Higaki, Jitsuo

Higashi, Yukihito

Hirano, Teruyuki

Hirano, Tsutomu

Hirashiki, Akihiro

Hirata, Kozo

Hirata, Yasunobu

Hirata, Yukio

Hirawa, Nobuhito

Hirohata, Satoshi

Hirooka, Yoshitaka

Hirose, Hiroshi

Hisatome, Ichiro

Hitomi, Hirofumi

Hocher, Berthold

Holowatz, Lacy

Horio, Takeshi

Horiuchi, Masatsugu

Horowitz, John

Hörtnagl, Heide

Hosomi, Naohisa

Hougaku, Hidetaka

Houston, Mark

Hozawa, Atsushi

Hróbjartsson, Asbjørn

Hsieh, Po-Shiuan

Huxley, Rachel

Hwang, Ki-Chul

Ichihara, Atsuhiro

Ichiki, Toshihiro

Igase, Michiya

Ikeda, Keiichi

Ikeda, Kyoji

Ikeda, Tomoaki

Iketani, Toshiro

Imai, Enyu

Imai, Yutaka

Inoguchi, Toyoshi

Inukai, Kouichi

Irita, Jun

Iseki, Kunitoshi

Ishikawa, Joji

Ishikawa, San-E

Ishikawa, Shizukiyo

Ishimitsu, Toshihiko

Ishimuara, Eiji

Ishizaka, Nobukazu

Itakura, Mitsuo

Ito, Hiroshi

Ito, Masaaki

Iwai, Masaru

Iwai, Naoharu

Iwao, Hiroshi

Iwasaki, Yasumasa

Izumi, Yoichi

Jaggar, Jonathan

Jellinger, Kurt

Johnson, John

Jordan, Jens
Kaaja, Risto

Kabutoya, Tomoyuki

Kageyama, Shigeru

Kai, Hisashi

Kamide, Kei

Kanno, Yoshihiko

Kario, Kazuomi

Karnik, Sadashiva

Kastrup, Jens

Kato, Norihiro

Katsuya, Tomohiro

Kawabe, Hiroshi

Kawada, Noritaka

Kawada, Toru

Kawakami, Masanobu

Kawano, Yuhei

Kayaba, Kazunori

Kazi, Tasneem

Kenmei, Takaichi

Kikuchi, Kenjiro

Kikuya, Masahiro

Kim, David

Kim, Jeongseon

Kimura, Akinori

Kimura, Genjiro

Kimura, Kenjiro

Kinae, Naohide

Kita, Toshihiro

Kitagawa, Kazuo

Kitakaze, Masafumi

Kitamura, Kazuo

Kiyomoto, Hideyasu

Kizu, Osamu

Kobayashi, Naohiko

Kobayashi, Yoshio

Kohara, Katsuhiko

Kokubo, Yoshihiro

Komai, Norio

Komuro, Issei

Kondo, Takahisa

Konoshita, Tadashi

Konta, Tsuneo

Koshiyama, Hiroyuki

Koya, Daisuke

Kraemer-Aguiar, Luiz Guilherme

Kramer, Holly

Kubo, Yutaka

Kumagai, Hiroo

Kurata, Akira

Kurata, Mie

Kureishi-Bando, Yasuko

Kuriyama, Satoru

Kurtz, Theodore

Kusano, Eiji

Kuwajima, Iwao

Kyotani, Shingo

Lee, Cheng-Han

Lee, Tony

Lee, Tsung-Ming

Leung, Ping-Chung

Li, Xiang-An
Lima-Costa, Fernanda

Lin, Yao-Ping

Linden, Wolfgang

Lindholt, Jes

Lindsey, Merry

Lotufo, Paulo

Madeddu, Paolo

Maemura, Koji

Mager, Ursula

Malamitsi-Puchner, Ariadne

Mansano, Roy

Maruyama, Shouichi

Masuda, Junichi

Masugata, Hisashi

Masuo, Kazuko

Masuyama, Hisashi

Masuyama, Tohru

Matsubara, Hiroaki

Matsubara, Mitsunobu

Matsuda, Hiroshi

Matsui, Hiromitsu

Matsui, Yoshio

Matsumae, Tomoji

Matsumoto, Yoshihiro

Matsumura, Yasuo

Matsuzaki, Masunori

May, Clive

Mayr, Winfried

Mcfadden, Christpher

Miki, Tetsuro

Millis, Richard

Minami, Junichi

Minamino, Tohru

Mitsuyama, Shokei

Miura, Katsuyuki

Miura, Shin-Ichiro

Miyakawa, Masaaki

Miyamori, Isamu

Miyata, Masaaki

Miyata, Toshiyuki

Miyauchi, Takashi

Mogi, Masaki

Momiyama, Yukihiko

Momomura, Shinichi

Mondo, Charles

Mori, Takefumi

Morimoto, Shigeto

Morishita, Ryuichi

Morita, Hiroyuki

Morita, Toshisuke

Morris, Mariana

Mukoyama, Masashi

Munakata, Masanori

Mune, Tomoatsu

Murakami, Yoshitaka

Murayama, Toshinori

Murohara, Toyoaki

Nabika, Toru

Nagase, Miki

Nagata, Daisuke

Nakagami, Hironori
Nakagami, Tomoko

Nakagawa, Takahiko

Nakagawara, Joji

Nakamoto, Hidetomo

Nakamura, Koshi

Nakamura, Satoko

Nakamura, Yasuyuki

Nakamura, Yoshikazu

Nakayama, Tomohiro

Nakazato, Masamitsu

Nakura, Jun

Nangaku, Masaomi

Nanjo, Kishio

Nara, Yasuo

Naruse, Katsuhiko

Naruse, Keiko

Naruse, Mitsuhide

Nichols, Wilmer

Ninomiya, Toshiharu

Nishikawa, Tetsuo

Nishikimi, Toshio

Nishinaga, Masanori

Nishiyama, Akira

Nitta, Kosaku

Noda, Akiko

Node, Koichi

O'Rourke, Michael F.

O'Sullivan, John

Oba, Koji

Ogawa, Hisao

Ogawa, Susumu

Ogawa, Tetsuya

Ogimoto, Akiyosi

Ohe, Tohru

Ohira, Tetsuya

Ohishi, Mitsuru

Ohkubo, Takayoshi

Ohkuchi, Akihide

Ohmori, Tsukasa

Ohte, Nobuyuki

Ohya, Yusuke

Ohyanagi, Mitsumasa

Oike, Yuichi

Oka, Tatsuzo

Okada, Tomohisa

Okada, Yasushi

Okamura, Tomonori

Okayama, Hideki

Oki, Kenji

Oki, Yutaka

Okumura, Ken

Okumura, Kenji

Okura, Takafumi

Oltman, Christine

Ono, Katsushige

Otsuka, Fumio

Otsuka, Kuniaki

Oyama, Yasuo

Parati, Gianfranco

Patel, Nimesh

Paunovic, Katarina 
Peng, Jun

Peter, Inga

Pimenta, Eduardo

Porter, James

Protogerou, Athanassios

Qiu, Changchun

Rajkumar, Chakravarthi

Rakugi, Hiromi

Reusz, György

Rigo Jr, Janos

Ris-Stalpers, Carrie

Rong, Zhou

Rosa, Ján

Rossi, Gian Paolo

Rubio-Guerra, Alberto

Ruilope, Luis

Rylander, Ragnar

Saavedra, Juan M

Saijo, Yasuaki

Saito, Ikuo

Saito, Isao

Saito, Yoshihiko

Saitoh, Shigeyuki

Salles, Gil

Sano, Motoaki

Sarafidis, Pantelis

Sasaki, Ken-Ichiro

Sasaki, Satoshi

Sasamura, Hiroyuki

Sata, Masataka

Sato, Atsuhisa

Satoh, Fumitoshi

Satoh, Noriko

Satoh, Toru

Schaffer, Jean

Schillaci, Giuseppe

Senbonmatsu, Takaaki

Senda, Shoichi

Seto, Shinji
Shibata, Hirotaka

Shimabukuro, Michio

Shimada, Kenei

Shimokawa, Hiroaki

Shimosawa, Tatsuo

Shimpo, Masahisa

Shindo, Takayuki

Shinohara, Yukito

Shiomi, Toshiaki

Shirai, Kohji

Shite, Junya

Sirtori, Cesare

Soardo, Giorgio

Solini, Anna

Soma, Masayoshi

Stergiou, George

Sugawara, Motoaki

Sugimoto, Ken

Surbek, Daniel

Suzuki, Etsu

Suzuki, Hiromichi

Tabara, Yasuharu

Tabuchi, Masaki

Takahashi, Masafumi

Takai, Shinji

Takaki, Akira

Takata, Yasunori

Takazawa, Kenji

Takeda, Kazuo

Takeda, Yoshiyu

Takeuchi, Kazuhisa

Tamaki, Toshiaki

Tamura, Kouichi

Tanahashi, Norio

Tanemoto, Masayuki

Terada, Yoshio

Terauchi, Yasuo

Terawaki, Hiroyuki

Thompson, Alex
Tikellis, Chris

Tocci, Giuliano

Tochikubo, Osamu

Tojo, Katsuyoshi

Tomita, Kimio

Tomita, Naruya

Tomiyama, Hirofumi

Torrens, Christopher

Toussaint, Nigel

Tseng, Chuen-Den

Tsioufis, Costas

Tsuchihashi, Takuya

Tsuji, Yukiomi

Tsutsui, Hiroyuki

Ueda, Shinichiro

Uehara, Yoshinari

Uehara, Yoshio

Ueshima, Hirotsugu

Ueyama, Takashi

Uezono, Keiko

Umemoto, Seiji

Umemura, Satoshi

Ura, Nobuyuki

Urata, Hidenori

Uzu, Takashi

Vaiman, Daniel

Van Den Born, Bert-Jan

Varon, Joseph

Velez, Juan Carlos

Vlachopoulos, Charalambos

Wakatsuki, Akihiko

Wang, Tao

Wang, Xingli

Wang, Zaimin

Wassmann, Sven

Watanabe, Hideki

Watanabe, Tsuyoshi

Webb, Clinton

Whaley-Connell, Adam
White, William

Wilkinson, Ian

Wu, Xiru

Xiao, Daliao

Yamada, Yuichi

Yamagishi, Masakazu

Yamagishi, Sho-Ichi

Yamaguchi, Osamu

Yamaguchi, Shuhei

Yamakado, Minoru

Yamamoto, Kazuhiro

Yamamoto, Tatsuo

Yamasaki, Fumiyasu

Yamashina, Akira

Yamashita, Takeshi

Yamashita, Tomoya

Yamazaki, Tsutomu

Yamori, Yukio

Yang, Wei-Shiung

Yano, Yuichiro

Yasuda, Osamu

Ye, Zhi-Bin

Yildiz, Mustafa

Yin, Xiaoyan

Yokoyama, Hitoshi

Yoshiaki, Taniyama

Yoshida, Masayuki

Yoshiji, Hitoshi

Yoshimoto, Takanobu

Yoshimura, Michihiro

Yoshizumi, Masanori

Yoshizumi, Masao

Zanchetti, Alberto

Zauli, Giorgio

Zhang, Yun

Zhao, Xueying

Zhou, Ziqiang 